\title{
Palm Print Recognition System using Naive Bayes Classifier and Image Processing Tools
}

\author{
Hosne-Al-Walid \\ Lecturer \\ Department of CSE \\ Ahsanullah \\ University of \\ Science and \\ Technology
}

\author{
Tasnia Sadia \\ Student \\ Department of CSE \\ Ahsanullah \\ University of \\ Science and \\ Technology
}

\author{
Anika Tahsin \\ Student \\ Department of CSE \\ Ahsanullah \\ University of \\ Science and \\ Technology
}

\author{
Tanzima Asad \\ Student \\ Department of CSE \\ Ahsanullah \\ University of \\ Science and \\ Technology
}

\author{
Nishat Tasnim \\ Student \\ Department of CSE \\ Ahsanullah \\ University of \\ Science and \\ Technology
}

\begin{abstract}
Biometric authentication has become a must in recent days. People have become part of many organization \& website where security maintenance is very important to ensure convenient system usability. Research regarding this issue is of a large significance to improve and increase performance of existing biometric system. In this particular paper it is tried to add some new features to existing palm print recognition system to check whether it enhances the authentication performance or not. One of the most important features of biometric system is that it does not need not to be memorized or kept safely. In the current era of technological advancement Biometric system is the ultimate solution regarding authentication \& identification process of tomorrow's world. In this system human palm has used for both ID \& Password which is uniquely used by the owner of that palm print only. The main and significant features of a palm print are extracted and stored in a database for enrollment and verification of human identification. A distinct feature of biometric system is that it can be used anywhere you want such as sign in a website, enter a building, attendance, bank account or financial security, security of highly classified information and where ever authentication of identity is required.
\end{abstract}

People are more vulnerable to security attack now. One can enter your private information without engaging much effort. Even most complex passwords cannot ensure $100 \%$ security. So, you need not to create different complex passwords and memorize them when you use a biometric system. Firstly images are preprocessed and the features are extracted to store them in the database to enroll the human. Because, the information should be available when you verify them using new data. Palm print provides a better performance for identification \& authentication compared to other biometric systems.

\section{General Terms}

Image Processing, Biometric System, Palm recognition, Naive Bayes Classifier

\section{Keywords}

Median Filter, Preprocessing, Threshold, Histogram, Distance Measure, Biometric System, Feature Extraction, Co-Ordinates of Key Points, Naive- Bayes Classifier

\section{INTRODUCTION}

To make security protocol more and more unbreakable is one of the main most difficult challenges of ensuring security at a higher rate to keep the desired system safe and convenient.
So, password with a large number of digits, case sensitivity and other regulations are applied to maintain security. These difficult passwords add more complexity to keep them in mind [1]. If people have many passwords which is very common such as log in password, bank account password, social network account password etc. it becomes nearly impossible to remember all of them. So system becomes inconvenient. Besides, if people write them to ease of use, it may be misused by wrong people which ultimately destroys the purpose of security measures. Privacy ensuring through password is thus become so difficult and personal data has gone much vulnerable now-a-days. PIN code has also the same disadvantages.ID cards are worse than passwords. People can use them though they do not belong to them. On the contrary, in biometric system physiological characteristics are used for identity verification such as finger prints, iris, face etc. [2] which can only be used by the people who actually own that particular body part. User does not need to memorize them and it is not possible to be used by other persons. Because of the uniqueness of physiological characteristics they are safer than other security measures. Even siblings, clones, twins have different physiological characteristics [3]. The unique physiological characteristics cannot be owned by two different people and cannot even changed or regenerated [4].

Image processing is always a very big challenge in machine learning as images of a same object are different at different times captured by the same machineries or image capturing sensors. It causes different values of co-ordinates and other measures. It becomes difficult in pattern matching to match these different values of same object. So you have to keep in mind the translation, rotation, scaling, reflection, occlusion and other factors while image pre-processing [5]. To achieve a good matching result from the system you have to keep this factors as lower as possible. Otherwise result may vary in large scale.

The first and probably the most challenging task is to have a clear and good image which is a must for the further steps of this system. For this purpose execute pre-processing and other procedure for extracting desired features and then store those features in a database [6]. When any person wants to enter the system it matches the persons instant extracted features (train data) with previously stored data (train data). Thus the verification of identity is performed.

There are several problems which may cause effect in the palm recognition. These are frame changing in each time, image angle taken, image size, image position etc. In the palm 
recognition, for matching these are great problems. For the purpose of image matching at first take an image, but then it may have noise or some other errors. These factors do not make the perfect image for the matching. Therefore it need to overcome these problems. The process to resolve the problems for matching of images is called pre-processing steps.

For matching the image pattern, there exist several algorithms. Palm recognition is different from them. At first check the features of palm and the values of those features are collected. Then as soon as it gets the image to match pattern, then for pattern matching purpose the images should be matched with the database.

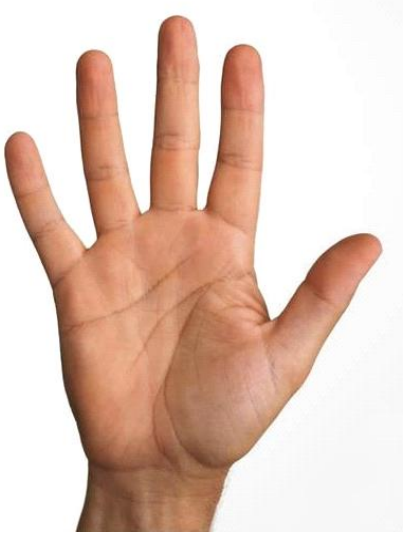

Figure 1: A palm image sample

\section{NECESSITIES FOR PALM PRINT TECHNOLOGY}

Now a days, identity proof is one of the most common methods in our daily life. For proving the identity of a person, the biometric of that person is used. There exits so many techniques in these sectors, like: Cards, thumb proof and so on [8]. So now it is the concern to develop a method in which result is with the good qualities, and almost error free while matching the image of palm [9]. The method should be cheap and easily used, time consuming and available. In this sector palm print recognition is one of the best techniques [10].

The palm print recognition is a unique and effective technique. As it can avoid the expensive devices for this technique, so it is very cost effective. Again the most important case is, hand is one of the body parts of every human being. Each man has different shape of palm of hand. So this palm of hand can easily be used a proof of one's identification, and also it can't be stolen.

There are so many reasons to prefer the palm images for the biometric applications. Like:

- Palm images vary from one to another.

- We can get more information from palm image than fingerprint.

- The images of palm can be easily captured and they are cost effective.

- The copy of those images is so difficult.

By the low resolution, the images of hand palm can be captured easily. As the other method like iris, card or finger print are so distinctive, so palm print is more useful here. By the palm images you can get many important information which are useful and effective to make the system perfect. Here images of hand palm are collected first then for matching purpose the values of those images are matched with the database.

There are many drawbacks exist in other techniques of identity recognition techniques. Like face recognition can be affected by age or other parts of human body. Again iris recognition is intrusive and expensive. Finger print is also expensive and we can get less information from this. So compared with all these techniques palm print recognition becomes more effective and useful day by day.

\section{PREPROCESSINGAND ACQUISITIONOF HAND IMAGE}

In palm recognition technique first you have to process the image of the palm. You can call it preprocessing of the image [11].This things have to be done to get more accurate, sharp image. Like first you have to store image in the database but when you store an image it may have many limitations. It may suffer from noise, shadows etc. For these reasons it needs to preprocess the image. Like preprocessing acquisition of hand image is also important. Acquisition is important because you need same size of the image each time. By this you can easily get the principle lines [12] and other features of the hand palm. In this paper most of the points of the hand palm are used. Here for palm recognition principle line distance, fingers top to center, fingers bottom to center, height, width, also length of the finger are used.

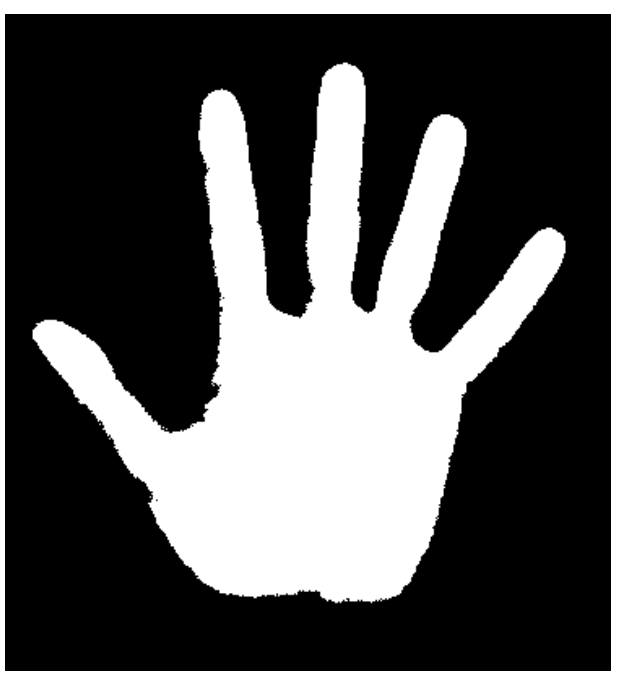

Figure 2: Background removal

First the salt and pepper noise is removed by filtering. After removing noise the image is seperated from the background [13]. That means the major part of the image is visible and background does not create any problem. The boundary of the palm is also detected. By detecting boundary of the palm the image is more visible to user. But for this you need to convert the image. After acquiring the image it converts the image. Here first it converted into RGB image into grey image then binary image [14]. 


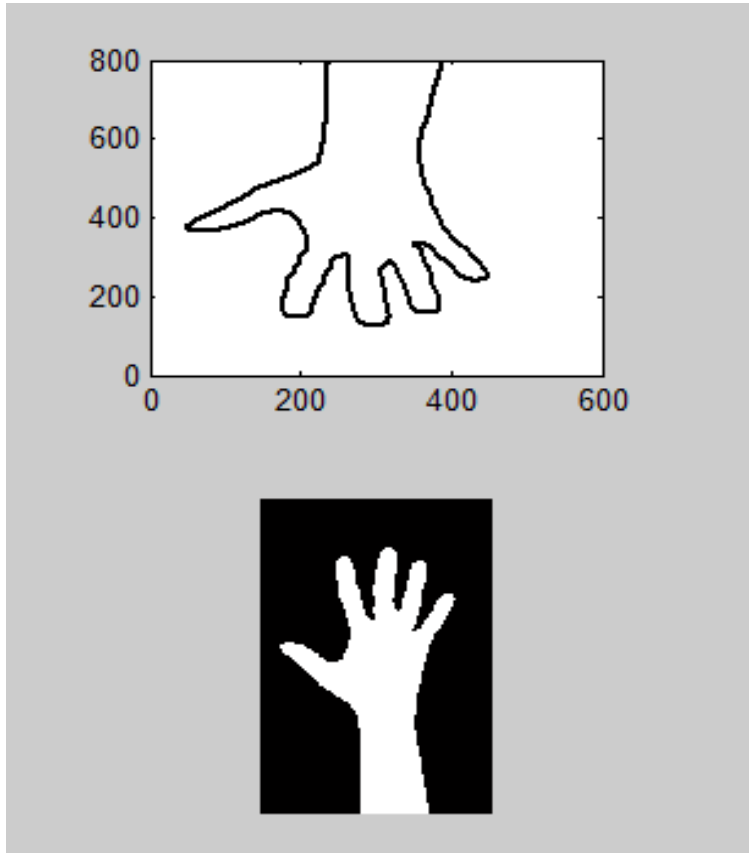

Figure 3: Boundary detection

And for converting the image into binary image it uses histogram analysis [16]. Here by using histogram it detects the local minimum points and then uses the points as threshold. It converts the pixels into black and white pixels. Using threshold we can easily convert the palm image into binary image [17]. After converting the image into binary image the palm image is ready for other operations.

In this palm print recognition technique it also uses border tracing algorithm. You can find the boundary of the palm image, position of fingers tips and some other features using border tracing algorithm [18].

\section{EXTRACT ROI AND OTHER PART OF PALM IMAGE}

\subsection{Extract the ROI}

In this paper the proposed system uses a square region of palm which is known as ROI (Region of Interest). For extracting ROI region it uses roipoly() function. Here a rectangular ROI region is used as there are various types of ROI region [19]. After detecting ROI region it is needed to detect principle lines of the image. For detecting principle lines there are many algorithms [20]. In this paper the system implements Sobel edge detection algorithm [21]. In binary image principle lines convert into white pixel and rest of the image convert into black pixels [22]

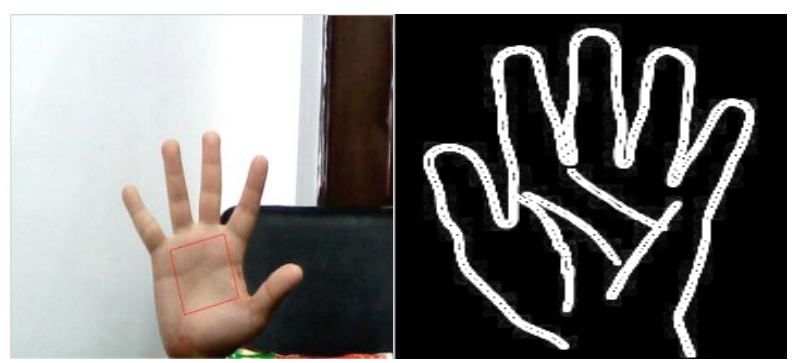

Figure 4: ROI region
Figure 5: Sobel edge detection

\subsection{Extract the other part of the palm image}

Lines of the fingers also converted into black and white pixels [23]. It uses root and top finger point as reference point of palm image. Here the system implements Discrete Cosine Transform (DCT) [24]. It stores the features of a palm image in database. And use these features in the verification process while matching it with a person's palm. In this paper we find the key points of hand palm including fingers.

\section{EXTRACT THE FEATURES OF THE PALM PRINT IMAGE}

In the palm recognition technique, the most important step you have to do is called feature extraction. The system used this process to get some important and meaningful features.

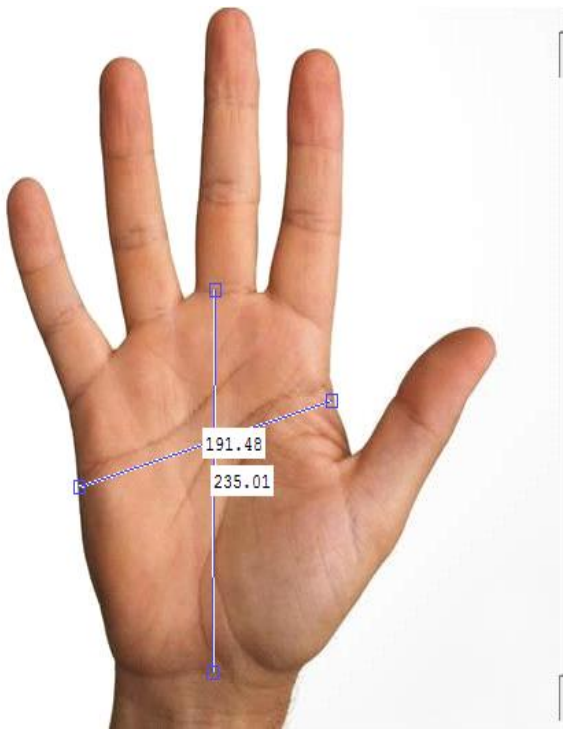

Figure 6: Height \& width of a palm image

Firstly detected the distance of principle lines in the ROI region. Secondly, it calculated the distance from center to top of the finger, center to bottom of the finger. After that it calculated the distance from bottom point to top point of fingers. This system has detected the height of the palm, width of the palm and length of the finger as well. There are many types of lines in the finger which varies from person to person. So you have to store the results in database which will help you regarding verification process of the image of the palm. The system has used Naïve-Bayes algorithm for classifying this. It has used this algorithm because it has most accuracy if you compare to other algorithms.

\section{OUR UNIQUE FEATURE}

While studying the previous papers on this topic \& it was found that most of the papers take in account the principle lines only. In one paper we found reference points and distance from those points are calculated [25]. In this system it added the top points of fingers and their distances from center of the ROI; height\& width of the palm and length of the fingers. This enhances some difficulties but efficiency can be improved.

\section{PSEUDOCODE}

Step-1: Remove noise from the acquired image.

Step-2: Distinguish the palm image from background. 
Step-3: Detect the boundary of the palm image.

Step-4: Convert the RGB image into Grey level \& then Binary level image.

Step-5: Get the histogram analysis of the acquired image using imhist() function.

Step-6: Use threshold to convert RGB pixels into black \& white pixels.

Step-7: Use border tracing algorithm to trace the border of palm image clearly.

Step-8: Extract ROI using roipoly() function.

Step-9: Use sobel edge detection algorithm to view the edges clearly.

Step-10: Use Discrete Cosine Transform (DCT) to detect and extract the principle lines.

Step-11: Find all the co-ordinates of required key points using imtool() function.

Step-12: Find the center of the ROI Using centroid() function.

Step-13: Measure all the required distances for the matching process using image viewer distance tool.

Step-14: Match the train data with test data using Naive Bayes classification algorithm.

Step-15: Show the classification result \& accuracy of the system.
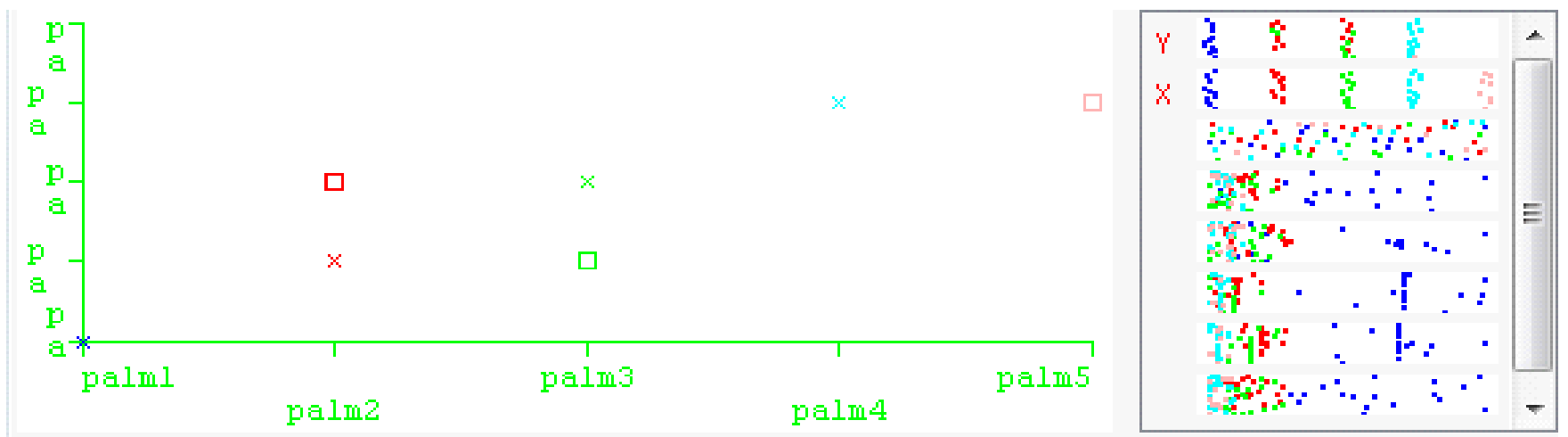

\section{RESULT ANALYSIS}

Here it used a data set of 100 instances. They include data from five different palm prints. It has taken principle lines length, fingers length, and distance from center to five top points of finger, distance from center to five bottom points of finger and height \& width of the palm. We classified the result:

$\begin{array}{llll}\text { Correctly Classified Instances } & 70 & 70 & \% \\ \text { Incorrectly Classified Instances } & 30 & 30 & \% \\ \text { Kappa statistic } & 0.625 & & \\ \text { Mean absolute error } & 0.1359 & \\ \text { Root mean squared error } & 0.2691 & \\ \text { Coverage of cases }(0.95 \text { level }) & 100 \quad \% & \\ \text { Mean rel. region size }(0.95 \text { level }) & 35 \quad \% & \end{array}$
instances with the help of Naive Bayes classifier. Here are the

Class colour

palm1 palm2 palm3 palm4 palm5

Figure 7: Classification figure

*squire points are correctly classified instances.

*cross points are incorrectly classified instances.

It is neither too good nor too bad. So It has a lot of challenges to meet to enhance the performance of the proposed system.

\section{CONCLUSION AND FUTURE WORK}

In this system it detected ROI, principle lines, center of the ROI, reference points \& required distances from those reference points. Then the extracted features of the whole palm is stored in the database during the enrollment process. While verification process, the system matches the stored data to newly acquired data. This is so far the most effective \& cheap biometric system for the authentication \& security purposes and not to carry anything along with us like card, code etc. for the verification purpose. There is a large scope to 


\section{REFERENCES}

[1] C. C. Han, H. L. Cheng, C. L. Lin, K. C. Fan, "PersonalAuthentication Using Palmprint Features", Pattern Recognition,Vol. 36, No. 2, pp. 371-381, 2003

[2] JoytiMalik,RatnaDahiya ,G.Sainaryanan , "Personal Authentication using Palm print with Phase Congruency feature Extraction method" , International journal of signal processing Image Processing and pattern Recognition vol.4 no.3 ,September 2011.

[3] Biometric Introduction, www.wikipedia.org/biometrics.

[4] K. Delac, M. Grgic, “A Survey of Biometric Recognition Methods", 46th International Symposium Electronics in Marine, ELMAR-2004, June 20[4] Biometric Introduction, www.wikipedia.org/biometrics.

[5] Chin-Chuan Han, Hsu-Liang Cheng, Chih-Lung Lin, Kuo- Chin Fan, "Personal authentication using palmprint features", Pattern Recognition 36 (2003) 371 - 381 , December 2011.

[6] I KetutGedeDarma Putra, Erdiawan, "High Performance Palmprint Identification System Based On Two Dimensional Gabor"TELKOMNIKA Vol. 8, No. 3, pp.309-318, 2010.

[7] CASIA Palmprint Database, http://biometrics.idealtest.org/dbDetailForUser.do?id=5

[8] L. Fang, M.K.H. Leung, T. Shikhare, V. Chan, K. F. Choon,"Palmprint Classification".

[9] SinaAkbariMistani, ShervinMinaee, EmadFatemizadeh, "Multispectral Palmprint Recognition Using a Hybrid Feature" ElectricalEngineering Department, Sharif University of Technology, Tehran, pp.1-7, 2011.

[10] PriyankaSomvanshi, MilindRane, "Survey of Palmprint Recognition", International Journal of Scientific \& Engineering Research, Vol. 3,pp.1-7, 2012.

[11] G.S. Lipane, S.B. Gundre, "Palm Print Recognition Review Paper", International journals srg , pp. 183-185,

[12] DewiYantiLiliana and Eries Tri Utaminingsih, "Thecombination of palm print and hand Geometry for biometricspalm recognition", International Journal of Video \&ImageProcessing and Network Security IJVIPNS-IJENS Vol: 12 No:01, feb 2012.

[13] R.C. Gonzalez, R.E. Woods, "Digital Image Processing UsingMATLAB", Second Edition, Pearson Education, 2002.7 .

[14] K.Y. Rajput, Mellisa Amanna, Mankhush Jagawat ,Mayank Sharma , "Palm print Recognition using Image
Processing",TECHNIA-International journal of computer science andcommunication technologies, vol.3,jan 2011(ISSN 0974-3375).

[15] IITD PalmprintDatabase, http://www4.comp.polyu.edu.hk/ csajaykr/IITD/Databas e_Palm.htm.

[16] Zhang W.K. Kong, J. You, M. Wong, "On-line palmprint identification", IEEE Transactions on Pattern Analysis and Machine Intelligence,Vol. 25 (9), pp.1041-1051, 2003.

[17] Abdul Ghafoor ,RaoNaveedIqbal and Shoiab Khan ,"Robust Image Matching Algorithm", Video/Image processing and multimedia communication , 5july,2003

[18] Wong K. Y. E., Jamal A. Dargham, Ali Chekima and G.Sainarayanan, "Palmprint Identification Using 5 x 5 Sobel Operator," Proceedings of First Seminar on Engineering andInformation Technology, 14-15 April 2008, Sabah Malaysia,Pages 208-211.

[19] N. Salman "Image Segmentation Based on Watershed and Edge Detection Techniques".

[20] D.K. Theckedath, "Image Processing Using MATLAB Codes Fourth Edition", Nandu Publications, 2009.

[21] Kong W.K., Zhang, D., Li W.X., "Palmprint feature extraction using 2-D Gabor filters." Pattern recognition, Vol. 36, pp.2339-2347, 2003.

[22] PatprapaTunkpien,SasipaPanduwadeethorn, SuphakantPhimoltares, "Compact Extraction of Principle Lines in Palmprint Using Consecutive Filtering Operations", Proceedings of the Second International Conference on Knowledge and Smart Technologies, pp.39-44, 2010

[23] Tee Connie, Andrew TeohBeng Jin, Michael GohKahOng, David Ngo Chek Ling, "An automated palmprint recognition system", Image andVisionComputing, Vol.23, pp.501-515, 2005

[24] Hafiz Imtiaz ,ShubhraAich ,ShaikhAnowarul Fattah "ANovalPre-processing Technique for DCT-domain Palm-printRecognition" ,International Journal of scientific \& TechnologyResearch ,volume 1 ,Issue 3.April 2012 ISSN 2277-8616.

[25] Jugal Kishore Patel,Sanjay Kumar Dubey,“ Deployment of Palm Recognition Approach using Image Processing Technique" ,International Journal of Computer Science Issues, Vol. 10, Issue 2, No 2, March 2013. 